\title{
MATRIX METHOD FOR DETERMINING THE INERTIA CHARACTERISTICS OF COMPOSITE ASYMMETRIC MECHANICAL SYSTEMS
}

\author{
D nipro U niversity of Technology \\ 19 D mytra Yavornytskoho Ave., Dnipro 49600, U kraine; e-mail: prof.w.kravets@ gmail.com
}

In this paper, the mathematical apparatus of monomial $(1,0 .-1) 4 \times 4$ matrices is applied to the development of an algorithm of inertia matrix transformation and the calculation of the center of mass of composite asymmetric vehicles as complex mechanical systems under translations and rotations of coordinate systems in space. Formulas for calculation are presented in harmonic, ordered, and compact matrix form directly adapted to computer technologies. The proposed new matrix algorithm allows one to effectively solve a wide range of problems of dynamic design of composite vehicles under substantial changes in the layout diagram both in structure and in composition. Vehicle layout diagrams are represented as complex spatial configurations in the form of individual subconstructions, which are asymmetric rigid bodies whose position and orientation in the layout diagram are varied in the course of the dynamic design of a complex mechanical system, for example, a launch vehicle. On the whole, a system of this type is considered as a composite asymmetric rigid body of complex spatial configuration. The dynamic performance of vehicles is mainly governed by their inertia characteristics, which include the total mass, the center of mass position, and the axial moment of inertia and the product of inertia calculated in a constructively convenient reference system. Vehicles of this type may be exemplified by hybrid motor vehicles, rail vehicles, flying vehicles of different purposes, etc.

The problems of motion stability, stabilization, steerability, and dynamic load are solved on the basis of a correct calculation of vehicle inertia characteristics. A correct calculation of inertia characteristics involves repeated spatial translations of the reduction centers of subconstructions and rotations of their axes, This complicated and cumbersome problem can be solved effectively by using the new mathematical apparatus of monomial (1, 0. -1) $4 \times 4$ matrices, which is conveniently implementable in computer technologies.

Keywords inertia characteristics, moments of inertia, center of mass, monomial matrices, quaternion matrices, Rodrigues-Hamilton parameters, vehicles.

1. Kravets V. V., Bass K. M., Kravets T. V., Tokar L. A. Dynamic design of ground transport with the help of computational experiment. MMSE Journal. 2015. V. 1. Pp. 105-111.

2. Kravets V. V., Bass K. M., Kravets T. V., Kharchenko A. V. Hybrid motor vehicle inertia matrix determination using quaternion matrices. System Technologies. 2012. No. 3 (80). Pp. 61-66. (in Russian).

3. Kharchenko A. V., Kravets T. V. Usage of quaternionic matrices to define inertia moments of wheel set taking into account manufacturing and assembly errors. 20th International Conference "Current Problems in Rail Vehicles", Zilina, September 21-23, 2011, Slovakia. Proceedings Nol. II. Pp. 109-114.

4. Alpatov A. P. Space Vehicle Dynamics. Kyiv: Naukova Dumka, 2016. 488 pp. (in Russian).

5. Kravets V. V. Evaluating the dynamic Load in a high-speed railroad car. Int. Appl. Mech. 2005. V. 41. No. 3. Pp. 324-329.

https://doi.org/10.1007/s10778-005-0091-7

6. Kravets V. V., Kravets T. V. On the nonlinear dynamics of elastically interacting asymmetric rigid bodies. Int. Appl. Mech. 2006. V. 42. V. 1. Pp. 110-114.

https://doi.org/10.1007/s10778-006-0065-4

7. Kravets V. V., Kravets T. V. Evaluation of the centrifugal, Coriolis, and gyroscopic forces on a railroad vehicle moving at high speed. Int. Appl. Mech. 2008. V. 44. No. 1. Pp. 101-109. https://doi.org/10.1007/s10778-008-0024-3

8. Kravets V., Kravets T., Burov O. Monomial (1, 0, -1)-Matrices-(4x4). Application to the Transfer in Space. Lap Lambert Academic Publishing, 2016. 137 pp.

9. Kravets V., Kravets T., Burov O. Monomial (1, 0, -1)-Matrices-(4x4). Part 2. Application to the Rotation in Space. Lap Lambert Academic Publishing, 2017. 82 pp. 
10. Kravets V., Kravets T., Burov O. Applying calculation of quaternionic matrices for formation of the tables of directional cosines. MMSE Journal. 2017. V. 11. Pp. 248-259.

Received on November 13, 2019 in final form on February 7, 2020 